\title{
SOLAR ROSSBY WAVE “HILLS” IDENTIFIED AS SUPERGRANULES
}

\author{
P. E. Williams, ${ }^{1}$ D. H. Hathaway, ${ }^{2}$ and M. Cuntz ${ }^{1}$ \\ Received 2007 January 31; accepted 2007 May 3; published 2007 June 8
}

\begin{abstract}
We explore the nature of "hills" observed on the solar surface that had previously been attributed to Rossby waves. We investigate the solar hills phenomenon by analyzing the output from a synthetic model based solely on the observed solar photospheric convection spectrum. We show that the characteristics of these hills can be explained by the corrugation of the surface produced by the radial flows of the convection. The hills in our simulations are dominated by supergranules, a well-known component of solar convection. Rossby waves have been predicted to exist within the Sun and may play an important role in the dynamics of the solar interior, including the Sun's differential rotation and magnetic dynamo. Our study suggests, however, that the hills observed at the solar limb do not confirm the existence of solar Rossby waves.
\end{abstract}

Subject headings: convection — Sun: helioseismology — Sun: photosphere — Sun: rotation — turbulence waves

\section{INTRODUCTION}

It is well known that the shape of the Sun is slightly nonspherical, its oblate shape exhibiting bulging at the equator and flattening at the poles due to rotation (e.g., Sofia et al. 1994). Observations using the Michelson Doppler Imager (MDI; Scherrer et al. 1995) and RHESSI instruments (Lin et al. 2002) both provide up-to-date evidence of this oblateness. More detailed studies of the collected data by Kuhn et al. $(1998,2000)$ reveal further variations in the shape of the solar limb, with evidence for small-scale "hills" on the surface. These hills have been characterized as being $100 \mathrm{~m}$ high and uniformly spaced by nearly $90,000 \mathrm{~km}$ over the solar surface and were thought to be a manifestation of solar Rossby waves (Kuhn et al. 2000).

Rossby waves (inertial waves whose restoring force is the latitude-dependent strength of the Coriolis force) were first proposed by Gilman (1969a, 1969b) to operate in the interior of the Sun. He suggested that these waves contribute to a magnetohydrodynamic dynamo that produces solar magnetic fields, including their periodic polarity reversals. Rossby waves could also be important in maintaining the structure of the Sun's differential rotation (see Kato \& Nakagawa 1969; Tikhomolov 1998; Wolff 1998). These theoretical studies suggest that Rossby waves may be connected to a large array of phenomena such as the solar surface structure, the Sun's magnetic cycle, the initiation and propagation of magnetic waves, and the dynamics of the solar atmospheric environment responsible for "space weather." It has been argued that similar effects also exist in other stars (Papaloizou \& Pringle 1978) and gaseous giant planets (Schaeffer \& Cardin 2005). Rossby waves were previously observed in the Earth's oceans (Chelton \& Schlax 1996; Cipollini et al. 2001) and atmosphere (Kursinski et al. 1996) as low-amplitude long wavelength features.

In their discovery of the Rossby wave hills, Kuhn et al. (2000) argued against the possibility that these hills were produced by supergranulation. Supergranulation is a photospheric convective phenomenon, consisting of cells typically $30,000 \mathrm{~km}$ in diameter that last for about a day (Leighton et al. 1962). Supergranulation cells are distributed fairly uniformly over the solar disk. Their

\footnotetext{
' Department of Physics, University of Texas at Arlington, Arlington, TX 76019-0059; pwilliams@uta.edu; cuntz@uta.edu.

${ }^{2}$ NASA Marshall Space Flight Center, Huntsville, AL 35812; david.hathaway@ nasa.gov.
}

boundaries are where much of the magnetic flux through the photosphere is concentrated-forming the magnetic foundation of the chromospheric network. On global scales, the transport of magnetic flux across the solar surface by supergranular flows plays a critical role in the evolution of the large-scale surface magnetic field. Such surface field patterns ultimately determine the photospheric footpoints of magnetic field lines as well as the chromospheric and coronal field topology, which is key for their nonradiative heating and dynamics. It has been found that the radial flow in typical supergranules is about $30 \mathrm{~m} \mathrm{~s}^{-1}$, corresponding to only $10 \%$ of their associated horizontal flows (Hathaway et al. 2000, 2002). These radial flows should produce a series of bumps on the surface of the Sun, but Kuhn et al. (2000) argue that the spacing would be too close and that the random forcing of these flows would not produce the spectral signatures they see.

In this Letter, we will examine the properties of hills on the solar surface produced by supergranules and show that these properties largely reproduce the spectral features attributed to Rossby waves by Kuhn et al. (2000). In $\S 2$, we describe the employed methods and results. Our conclusions are given in $\S 3$.

\section{METHODS AND RESULTS}

We take the following approach. Photospheric velocity data are produced using the method described by Hathaway (1988) that is based on the construction of synthetic data that realistically match observed photospheric velocity fields (Fig. 1). These simulations have successfully been used in previous studies (Hathaway et al. 2000, 2002, 2006). The radial component of the velocity field produces variations in photospheric heights that are then analyzed in a manner equivalent to that employed by Kuhn et al. (2000) in their analysis of the solar hills seen at the limb.

The limb signal data are produced from simulated patterns of the radial velocities on the solar disk following the method of Hathaway (1988). The radial velocities are generated by spectral coefficients $R_{\ell m}$ and the spherical harmonics $Y_{\ell m}(\theta$, $\phi)$. The magnitudes of the spectral coefficients are obtained by matching the Doppler velocity patterns observed from the MDI instrument on the ESA/NASA Solar and Heliospheric Observatory $(\mathrm{SOHO})$ Mission with the Doppler velocity patterns produced in the simulations (Hathaway et al. 2000, 2002). A simplified photospheric convection spectrum can match these data 


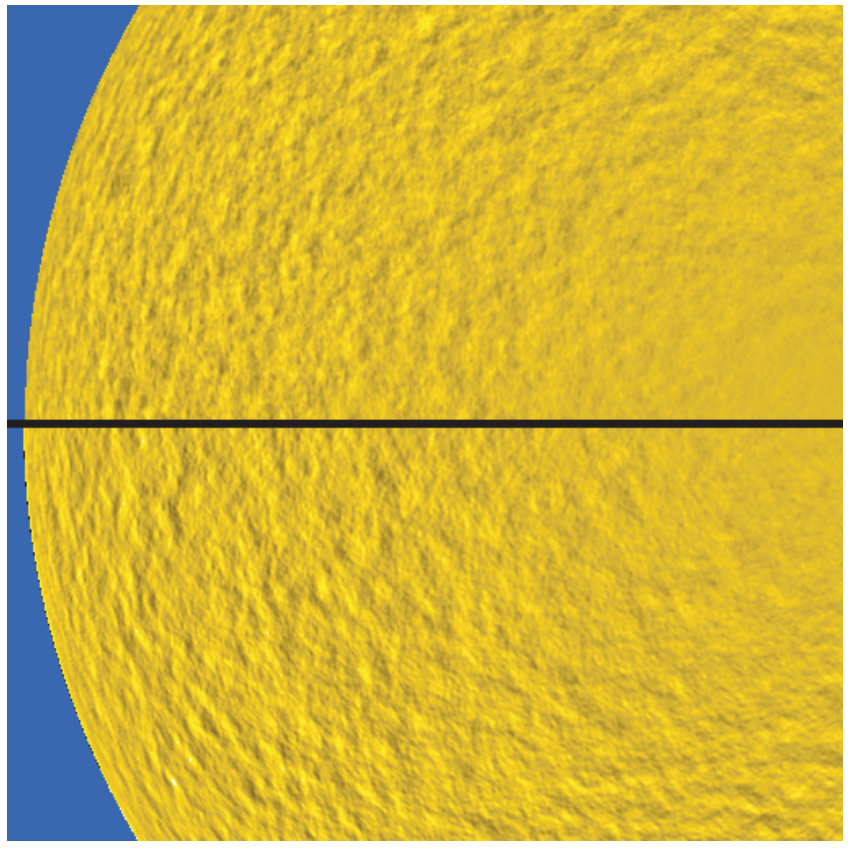

FIG. 1.-Comparison of Dopplergrams produced from $\mathrm{SOHO}$ MDI observed data (top) and simulated data (bottom). Bright features are redshifted (receding) and dark features are blueshifted (approaching). The similarity is apparent as are the surface features due to supergranulation.

over a wavenumber range of $\ell \sim 1-1000$ using two Lorentzian profiles, one for supergranules with a peak at $\ell \sim 110$ and another for granules with a peak at $\ell \sim 4000$. The parameters used to construct these component spectral profiles are adjusted to attain a close match between the simulated and observed photospheric convection spectra (Fig. 2).

The spectral coefficients for the radial velocity are converted to spectral coefficients for height by dividing the velocity amplitude by the square root of the wavenumber. This relation is adapted from the theory for up-flowing gas forming a "forced plume" subject to buoyancy with mass flux and momentum conservation (Turner 1973). Realistic numerical models for solar granulation (Stein \& Nordlund 1998) indicate that these smaller convective features have diameters of $\sim 1000 \mathrm{~km}$, radial velocities of $\sim 3000 \mathrm{~m} \mathrm{~s}^{-1}$, and produce surface height variations of $\sim 30 \mathrm{~km}$. These granular parameters can be scaled using the forced plume model to determine supergranule height variations, an operation derived from mass conservation. Using the characteristic size $(30,000 \mathrm{~km})$ and radial velocities $\left(30 \mathrm{~m} \mathrm{~s}^{-1}\right)$ for supergranules indicates that supergranules should produce hills with heights of $\sim 2 \mathrm{~km}$. This is more than an order of magnitude larger than the estimate reported by Kuhn et al. (2000) and may reflect on differences in driving mechanisims for granules and supergranules. The actual size of the hills is not what is important here. The spectral features identified by Kuhn et al. (2000) are temporal signatures associated with the rate at which these hills rotate into view over the limb.

We produce height maps of $4096 \times 1024$ elements (longitude by latitude), representing the full solar surface in a similar manner to how the Doppler velocity maps had been produced in previous studies (Hathaway 1988; Hathaway et al. 2000,2002 , 2006). Two latitudinal pixel columns separated by 2048 longitude pixels (i.e., meridional columns separated by $180^{\circ}$ in longitude) are extracted from the height map array.

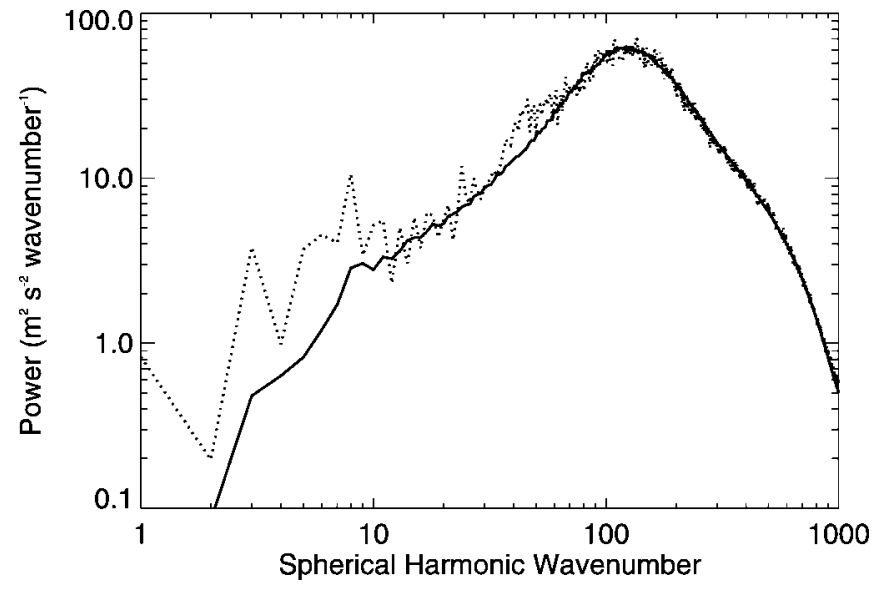

FIG. 2.-Comparison between the observed photospheric convection spectrum (solid line) and that for the simulation (dotted line) created from granule and supergranule components. The spectra match exceedingly well for wavenumbers $\ell$ from 10 to 1000 . The differences at the lowest wavenumbers are attributed to uncertainties in the instrumental artifacts found in the SOHO MDI data. The peak at wavenumbers near 110 is due to supergranulation.

These columns are reallocated into a single one-dimensional array representing the limb height all the way around the Sun starting from the west (as seen in the sky) and proceeding counterclockwise through north. The center of the right-hand column represents the western limb, and the data north of that represent the northwestern limb (ending at the north pole). The data from the left-hand column are joined on (north to south) representing the eastern limb; then the remainder of the first column is joined onto that (south pole to western limb). This is repeated for each time step (a solar rotation/4096) by stepping through the entire height map one longitude step at a time. (Note that halfway through the extraction, the western limb data column wraps around back to the far left of the map.) These limb height strips are combined into a two-dimensional array, so they represent a series of limb height data around the solar limb for each 9 minute time interval, or conversely, a series of limb height data at particular positions around the limb over one whole 27 day rotation sampled at a cadence of 9 minutes. A set of 30 such 27 day time series are produced, analyzed, and averaged for the purposes of noise reduction. Note that these simulations do not include differential rotation-the patterns rotate rigidly.

Additional data sets are produced representing limb heights over 34 days for evolving supergranule patterns. The patterns are evolved by adding random changes in phase to the spectral coefficients, while holding the amplitudes fixed, and accumulating these over time. Applying the same cross-correlation methods employed by Duvall (1980) and Snodgrass \& Ulrich (1990) to the simulated data produces supergranule lifetimes on the order of a day, coinciding with what is seen in solar data (Duvall 1980), and thereby removing any long-range coherence in the time series. These data are then reformatted into 34 day time series with a 12 minute cadence (matching the cadence used by Kuhn et al. 2000).

Each limb scan strip is convolved with a 4 pixel wide filter and the data sampled every fourth pixel, to create $512 \mathrm{limb}$ position bins similar to those produced by Kuhn et al. (2000). Taking the fast Fourier transform (FFT) of each of these 512 pixel spatial strips produces a spatial spectrum for each time sample. Subsequent sampling along the time strips and taking the FFT of each strip similarly produces a temporal spectrum 


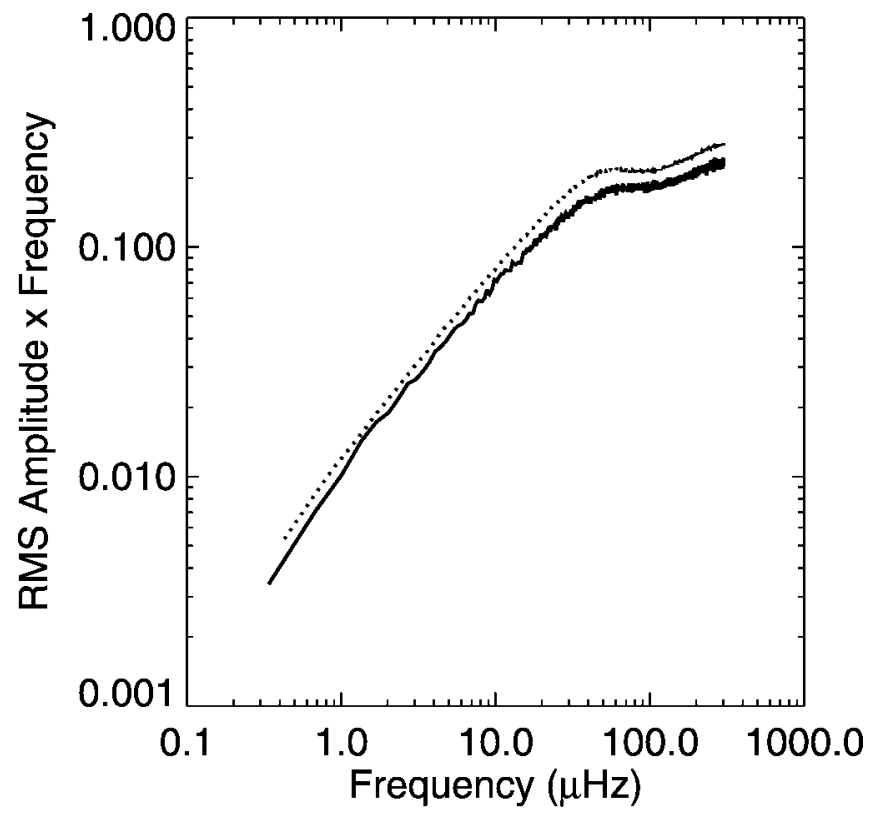

FIG. 3.-Power spectrum created by taking the spatial and temporal FFTs of the limb height time series summed over wavenumber $\ell$ and rescaled to similarly represent the plot constructed by Kuhn et al. (2000). The solid line is the average from three evolving supergranule simulations. The dotted line is the average from 30 nonevolving supergranule simulations. The excess of signal seen as a bump at a frequency of around $50 \mu \mathrm{Hz}$ is in good agreement with, but somewhat higher than, that seen by Kuhn et al. (2000).

for each spatial frequency. This results in two-dimensional arrays that are the two-dimensional (2D) FFT of the original spatiotemporal data. Multiplying these 2D FFT arrays by their complex conjugate gives power spectra for the limb height over a range of spatial and temporal frequencies. The power spectra are

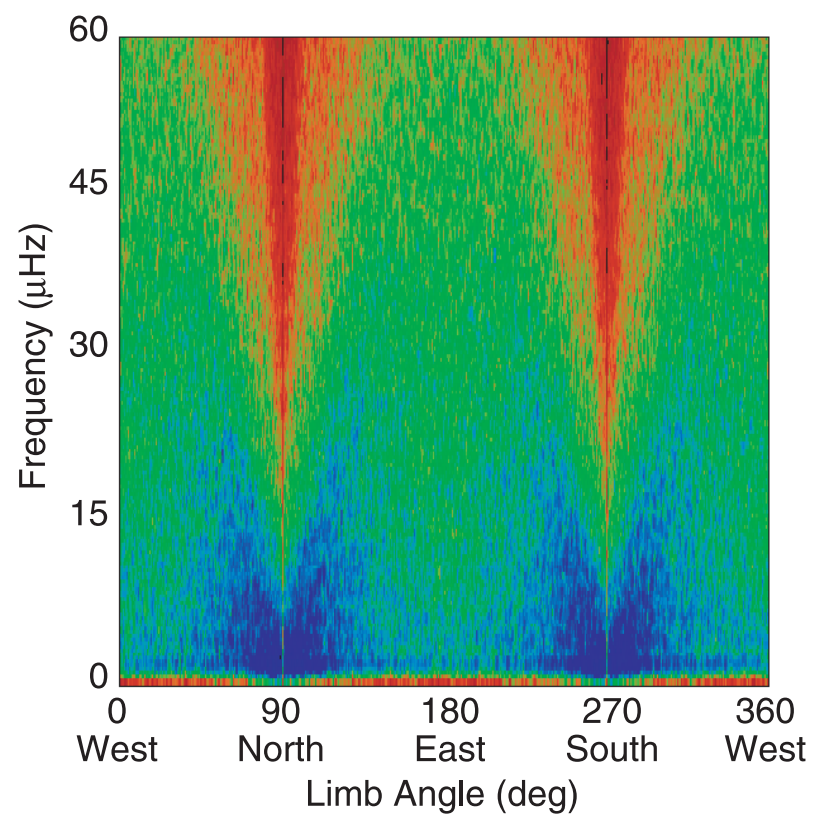

FIG. 4.-Average power spectrum from the three evolving flow pattern data for a range of temporal frequencies at each angular position around the limb. The signal strength increases logarithmically as color changes from red to green to blue. The result is a near sawtooth distribution of excess power, similar to that found by Kuhn et al. (2000). This illustrates that by distributing similarly sized features over a rotating sphere, features at the equator are more numerous and pass over the limb more frequently than at the poles.

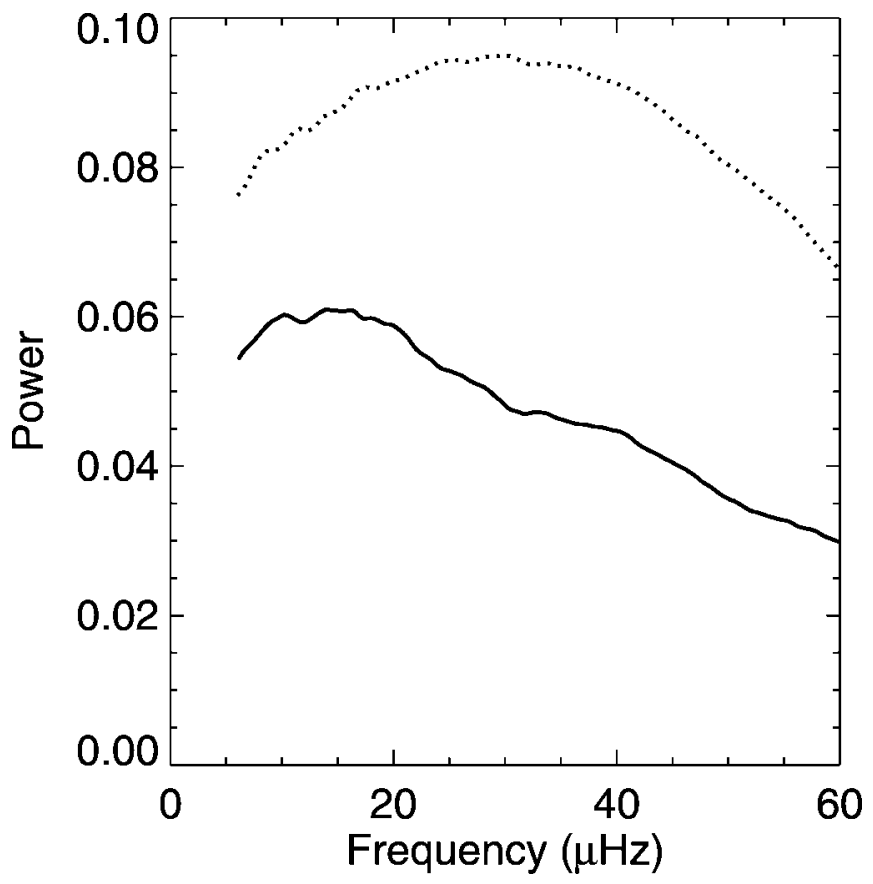

FIG. 5.-Spectral data from which Fig. 4 is produced are stretched for each limb position in proportion to its linear rotation velocity relative to that of the equator. Those data are then averaged over each temporal frequency bin and smoothed. The results for the 30 nonevolving supergranule simulations display a peak near $30 \mu \mathrm{Hz}$ as shown by the dotted line. The results for the three evolving supergranule simulations display a peak near $15 \mu \mathrm{Hz}$ as shown by the solid line. This spectral peak is similar to that found by Kuhn et al. (2000) from $S O H O$ MDI data.

summed over all spatial frequencies $(\ell=1-255)$ and then multiplied by the temporal frequency to produce the spectra shown in Figure 3. It is clearly seen that an excess of power is present at a frequency of $\sim 50 \mu \mathrm{Hz}$ (slightly higher than the frequency of the peak seen by Kuhn et al. 2000). This feature is produced by the supergranules in our simulation. The smaller granules are not well resolved in $\mathrm{SOHO} \mathrm{MDI}$ data as the data are further smoothed and resampled to get data at 512 limb positions.

The simulated data can also be used to produce temporal spectra of the limb signal as a function of limb position angle using a process similar to that of Kuhn et al. (2000). The data are smoothed in the spatial direction with a 256 bin running mean and in the temporal direction with an 8.3 day running mean, and the residual between the smoothed and original data calculated. The temporal FFT of this residual power spectrum is taken at each limb position and smoothed with a 4 pixel bin running mean for the 27 (or 34) day data stream (corresponding to the 88 pixel bin running mean over the 800 day sequence used by Kuhn et al. 2000). Multiplying this result with its complex conjugate produces temporal power spectra that can be plotted against position angle, as shown in Figure 4. A "sawtooth" pattern is seen, similar to that produced by Kuhn et al. (2000) with the maximum and minimum frequencies occurring at the equator and the poles, respectively.

The temporal power spectra at each limb position are stretched in proportion to the linear speed of rotation at the corresponding latitudes so that the maximum for each limb angle now lies along the frequency of maximum power at the solar equator instead of distributed along the sawtooth pattern. The power spectra are then averaged over all limb angles and plotted against the temporal frequency (Fig. 5). The nonevolving supergranule patterns give a peak near $30 \mu \mathrm{Hz}$, while the 
evolving supergranule patterns have reduced power with a peak near $15 \mu \mathrm{Hz}$-almost identical to the frequency obtained by Kuhn et al. (2000).

\section{CONCLUSIONS}

In this Letter, we explored the nature of "hills" observed on the solar surface that had previously been attributed to Rossby waves. The solar hills phenomenon is investigated by analyzing the output from a synthetic model based solely on the observed solar photospheric convection spectrum (Hathaway et al. 2000, 2002). We show that the characteristics of these hills can largely be reproduced by the corrugation of the surface due to the radial flows of the convection. The hills in our simulations are dominated by supergranules, a well-known component of the solar convection spectrum. This result is in disagreement with the conclusion by Kuhn et al. (2000) that these spectral features cannot be produced by supergranules. Although the $2 \mathrm{~km}$ heights we derived by means of mass conservation within a forced plume model may be an overestimation owing to the model's simplicity, the observed spectral features presented in Figures 3-5 are independent of the actual height of the hills and closely match the spectral features seen by Kuhn et al. (2000). The small differences in the shapes and positions of the spectral features are probably due to simplifications in our model. We do not include differential rotation of the pattern and the method used to evolve the pattern is purely statistical without regard for the surrounding flows or magnetic elements. The signatures that lead to our conclusions are produced from a spherical harmonic simulation of the photospheric Doppler velocity field based on a convection spectrum containing only supergranule and granule components. No wave phenomena whatsoever are included.

We argue that what have been detected are hills due to supergranulation. Our model was run for three scenarios, both spectral components, as described above, granulation only and supergranulation only. On removing the supergranulation, it is found that despite the spectral overlap of the supergranule and granule components, the excess power features as seen in Figures 3 and 5 disappear; thus, granulation cannot be responsible for the observed signal as expected from the small size of these features. However, the feature reappears for the spectrum containing only the supergranulation component. Thus, we conclude that the signal previously attributed to Rossby waves is most likely due to supergranulation. On comparing our results to the observations of Kuhn et al. (2000) we envision that the latter have provided an independent technique for characterizing supergranules, which may harbor future research potential.

In a recent paper, Kaladze \& Wu (2006) used the results of Kuhn et al. (2000) to explain the hill phenomena in the context of Rossby waves. As these observations can now be explained by supergranulation, it can be concluded that Kaladze \& Wu attempted to base their model on what turns out to be supergranule features. It is conceivable that what their model describes are indeed underlying features not yet detected on the Sun, but at most this work is deemed speculative and not backed up by unequivocal observational data.

Direct observational evidence of solar Rossby waves are still yet to be confirmed. While our work presented in this Letter concludes that the signals reported by Kuhn et al. (2000) are explained by supergranulation, there may yet be Rossby wave signatures still hidden within the observational data reported by Kuhn et al. (1998). Data collected by the RHESSI instrument (Lin et al. 2002) as reported by Fivian et al. (2005) may also contain Rossby wave signatures. Both sets of data, however, would require the necessary filtering, including the removal of the supergranulation signal, to extract any signals pertaining to Rossby waves.

This work was supported by NASA's Office of Space Science through a grant from its Solar and Heliospheric Supporting Research and Technology Program.

\section{REFERENCES}

Chelton, D. B., \& Schlax, M. G. 1996, Science, 272, 234

Cipollini, P., Cromwell, D., Challenor, P. G., \& Raffaglio, S. 2001, Geophys. Res. Let., 28, 323

Duvall, T. L. 1980, Sol. Phys., 66, 213

Fivian, M. D., Hudson, H. S., \& Lin, R. P. 2005, in The Dynamic Sun: Challenges for Theory and Observations, ed. D. Danesy et al. (ESA SP600; Noordwijk: ESA), CD-ROM 4.1

Gilman, P. A. 1969a, Sol. Phys., 8, 316 1969b, Sol. Phys., 9, 3

Hathaway, D. H. 1988, Sol. Phys., 117, 329

Hathaway, D. H., Beck, J. G., Bogart, R. S., Bachmann, K. T., Khatri, G., Petitto, J. M., Han, S., \& Raymond, J. 2000, Sol. Phys., 193, 299

Hathaway, D. H., Beck, J. G., Han, S., \& Raymond, J. 2002, Sol. Phys., 205, 25

Hathaway, D. H., Williams, P. E., \& Cuntz, M. 2006, ApJ, 644, 598

Kaladze, T. D., \& Wu, D. J. 2006, ApJ, 638, L49
Kato, S., \& Nakagawa, Y. 1969, Sol. Phys., 10, 476

Kuhn, J. R., Armstrong, J. D., Bush, R. I., \& Scherrer, P. 2000, Nature, 405, 544

Kuhn, J. R., Bush, R. I., Scheick, X., \& Scherrer, P. 1998, Nature, 392, 155

Kursinski, E. R., et al. 1996, Science, 271, 1107

Leighton, R. B., Noyes, R. W., \& Simon, G. W. 1962, ApJ, 135, 474

Lin, R. P., et al. 2002, Sol. Phys., 210, 3

Papaloizou, J., \& Pringle, J. E. 1978, MNRAS, 182, 423

Schaeffer, N., \& Cardin, P. 2005, Nonlinear Processes in Geophys., 12, 947

Scherrer, P. H., et al. 1995, Sol. Phys., 162, 129

Snodgrass, H. B., \& Ulrich, R. K. 1990, ApJ, 351, 309

Sofia, S., Heaps, W., \& Twigg, L. W. 1994, ApJ, 427, 1048

Stein, R. F., \& Nordlund, Å. 1998, ApJ, 499, 914

Tikhomolov, E. 1998, ApJ, 499, 905

Turner, J. S. 1973, Buoyancy Effects in Fluids (Cambridge: Cambridge Univ. Press)

Wolff, C. L. 1998, ApJ, 502, 961 